\title{
Revisiting the Hunter diagram with the Geneva Stellar Evolution Code
}

\author{
R. Simoniello ${ }^{1}$, G. Meynet ${ }^{2}$, S. Ekström ${ }^{2}$, C. Georgy ${ }^{3}$ and \\ A. Granada ${ }^{2}$ \\ ${ }^{1}$ Laboratoire AIM, CEA/DSM-CNRS-Université Paris Diderot; CEA, IRFU, SAp, centre de \\ Saclay, F-91191, Gif-sur-Yvette, France \\ email: rosaria.simoniello@cea.fr \\ ${ }^{2}$ Geneva Observatory, Chemin de Maillettes 51, 1290 Sauverny, Switzerland \\ ${ }^{3}$ Astrophysics group, Lennard-Jones Laboratories, EPSAM, Keele University, Staffordshire \\ ST5 5BG, UK
}

\begin{abstract}
We produced a model grid of rotating main and post-main sequence stars with the Geneva Stellar Evolution Code (GENEC). The initial chemical composition is tailored to compare with observations of early OB type stars in the Large Magellanic Cloud (LMC) and the grid covers stellar masses in the range of $7 \leqslant M / M_{\odot} \leqslant 15$ and initial velocity between $0 \mathrm{~km} \mathrm{~s}^{-1} \leqslant v \sin (i) \leqslant 300 \mathrm{~km} \mathrm{~s}^{-1}$. The model grid has been used to determine the changes in the surface Nitrogen abundances during the star evolution and the results have been compared with observations.
\end{abstract}

Keywords. methods: numerical, stars: rotation, stars: abundances

\section{Introduction}

The rotating evolutionary models for single stars predict that only the fastest rotating stars can be highly enriched in nitrogen (N) while still on the MS. Therefore a particularly strong observational diagnostic for rotational mixing is the $\mathrm{N}$ surface abundance of stars undergoing hydrogen fusion through the CNO cycle. A survey of massive stars has been carried out by the Very Large Telescope (VLT) Fiber Large Array Multi-Element Spectrograph (FLAMES) including $750 \mathrm{O}$ and early B type on clusters located in our Galaxy, the Large (LMC) and Small Magellanic Clouds (SMC). A detailed analysis of the relationship between $\mathrm{N}$ abundances and the projected rotational velocity $(v \sin i)$ has been carried out for early B type stars. The distribution of stars is called the Hunter diagram and it became one of the key diagrams to check the physics of rotational mixing (Hunter et al. 2008). It turned out that the current rotating models failed to explain the existence of highly $\mathrm{N}$ enriched slow rotators. This lead to intense debates on the unknown causes of the discrepancies and on the identification of other physical mechanisms or ingredients still missing in the description of the theoretical models. Within this context we decided to investigate the efficiency of the rotational mixing on Nitrogen surface abundances in an attempt to quantify the discrepancy with observations.

\section{Data analysis}

\subsection{The model grids}

We used GENeva stellar Evolution Code (GENEC), a one dimensional hydrodynamic code (Eggenberger et al. 2008), for the computation of new grids of stellar rotating models for masses between $5 \leqslant M / M_{\odot} \leqslant 15$, initial velocity between $0 \leqslant v \sin (i) \leqslant 300 \mathrm{~km} \mathrm{~s}^{-1}$ 


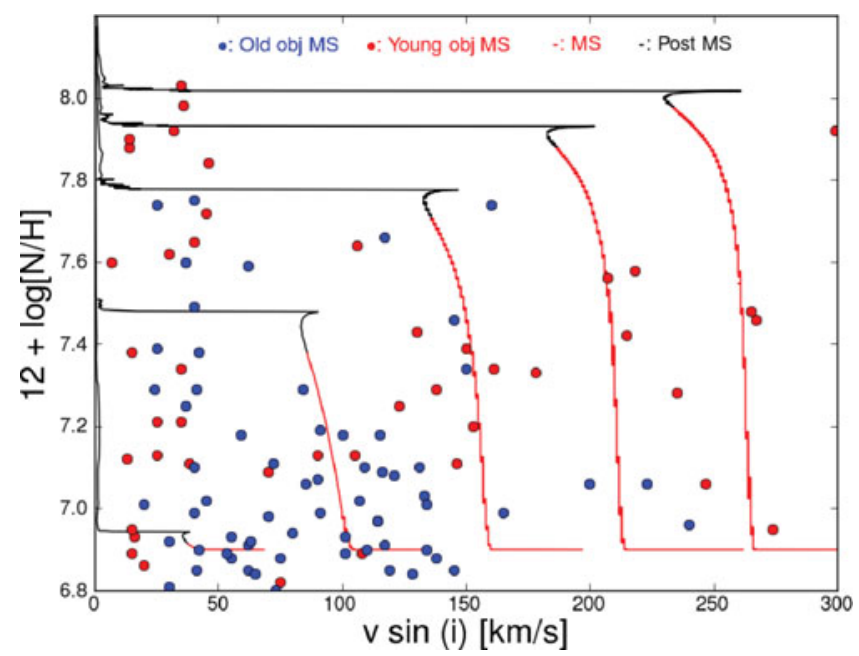

Figure 1. Nitrogen abundance against rotational velocity at LMC metallicity for rotating models at $12 M_{\odot}$ (continuous lines) and from observations of the VLT (bullet).

and with initial chemical composition $Z=0.0047$ matching that observed for the LMC, from which the richest massive star data are obtained.

\subsection{Results}

Figure 1 shows the N surface abundances as function of the projected rotational velocity. The blue and red dots represent the observed sample of early OB type stars in the N11 and NGC2004 LMC cloud downloaded from http://vizier.u-strasbg.fr/viz-bin/ VizieR-4 (Hunter et al. 2008). They have been split in two groups: young (surface gravity $\log g \geqslant 3.7)$ and old $(\log g \leqslant 3.2)$ core hydrogen burning objects. The red and black continuous lines represent the amount of nitrogen surface abundances during the evolution for two different evolutionary phase: main and post main sequence stars. The nitrogen abundance is for a $12 M_{\odot}$ and for different initial velocity $0 \leqslant v \sin (i) \leqslant 300 \mathrm{~km} \mathrm{~s}^{-1}$. As we can see the slowly rotating $\left(\leqslant 50 \mathrm{~km} \mathrm{~s}^{-1}\right)$ and highly nitrogen enriched $(\geqslant 7.2)$ young objects fall off the corresponding young branch of the evolutionary track, while young objects and old but slowly rotating still on the MS can be reproduced by the current rotating models.

\section{Conclusion}

This model grid constitutes the basis to producing a stellar population synthesis with the SYCLIST code (Georgy et al. 2014). This will allow us to get a statistically significant sample covering a specific range of masses and velocities and its comparison with the observed Hunter diagram will tell us the measure of the departure of the theoretical models from the observations.

\section{References}

Eggenberger, P., Meynet, G., Maeder, A., et al. 2008, APSS 316, 43

Georgy, C., Granada, A., Ekström, S., et al. 2014, A $₫ A$ 566, A21

Hunter, I., Brott, I., Lennon, D. J., et al. 2008, ApJL 676, L29 